\title{
Integrating Palliative Care in Pediatric Oncology: Evidence for an Evolving Paradigm for Comprehensive Cancer Care
}

\author{
Deena R. Levine, MDª Liza-Marie Johnson, MD, MPH, MSB'a; Angela Snyder, MSNa; \\ Robert K. Wiser, MBA ${ }^{\text {; }}$ Deborah Gibson, MAª Javier R. Kane, MDc; and Justin N. Baker, MD ${ }^{a}$
}

\begin{abstract}
Background: The demonstrated benefit of integrating palliative care (PC) into cancer treatment has triggered an increased need for PC services. The trajectory of integrating PC in comprehensive cancer centers, particularly pediatric centers, is unknown. We describe our 8-year experience of initiating and establishing PC with the Quality of Life Service (QoLS) at St. Jude Children's Research Hospital. Methods: We retrospectively reviewed records of patients seen by the QoLS ( $n=615)$ from March 2007 to December 2014. Variables analyzed for each year, using descriptive statistics, included diagnostic groups, QoLS encounters, goals of care, duration of survival, and location of death. Results: Total QoLS patient encounters increased from 58 (2007) to 1,297 (2014), new consults increased from 17 (2007) to 115 (2014), and mean encounters per patient increased from 5.06 (2007) to 16.11 (2014). Goal of care at initial consultation shifted from primarily comfort to an increasing goal of cure. The median number of days from initial consult to death increased from 52 days (2008) to 223 days (2014). A trend toward increased outpatient location of death was noted with $42 \%$ outpatient deaths in 2007 , increasing to a majority in each subsequent year (range, 51\%-74\%). Hospital-wide, patients receiving PC services before death increased from approximately $50 \%$ to nearly $100 \%$. Conclusions: Since its inception, the QoLS experienced a dramatic increase in referrals and encounters per patient, increased use by all clinical services, a trend toward earlier consultation and longer term follow-up, increasing outpatient location of death, and nearuniversal PC involvement at the end-of-life. The successful integration of PC in a comprehensive cancer center, and the resulting potential for improved care provision over time, can serve as a model for other programs on a broad scale.
\end{abstract}

J Natl Compr Canc Netw 2016;14(6):741-748

\section{Background}

Pediatric palliative care (PPC) is "aimed at enhancing quality of life, promoting healing, and attending to suffering in children" 1 affected by life-limiting, chronic, or life-threatening illness. ${ }^{2-4}$ The movement to formally develop high-quality palliative care (PC) programs tailored to children and their families began in the late 1990s. ${ }^{5-10}$ The American Academy of Pediatrics (AAP) issued the first policy statement on PPC in 2000, recommending that "all general and subspecialty pediatricians, family physicians, pain specialists, and pediatric surgeons need to become familiar and comfortable with the provision of PC to children." ${ }^{11}$ In 2003 the IOM recommended spe-

From the a Department of Oncology, Division of Quality of Life and Palliative Care, St. Jude Children's Research Hospital, Memphis, Tennessee; bUniversity of Tennessee Health Science Center, Memphis, Tennessee; and 'Department of Pediatric Hematology Oncology, McLane Children's Scott and White Clinic, Texas A\&M Health Science Center College of Medicine, Temple, Texas.

Submitted November 10, 2015; accepted for publication March 7, 2016. cialty training in PPC along with the development of collaborative guidelines and protocols tailored to children. ${ }^{12}$

In response to the priority areas set by the AAP and IOM, St. Jude Children's Research Hospital (St. Jude) identified the formation of a PPC program as an institutional priority in 2006,13,14 with the mission to "provide each child living with or dying from a catastrophic illness with state-of-the-art patient and family-centered physical, emotional, and spiritual care with the goal to attend to suffering, promote healing, and improve quality of life."15

In March 2007, St. Jude initiated a pilot project to evaluate the incorporation of PPC expertise as part of the interdisciplinary care team, with the goal of im-

The authors have disclosed that they have no financial interests, arrangements, affiliations, or commercial interests with the manufacturers of any products discussed in this article or their competitors.

Correspondence: Justin N. Baker, MD, Division of Quality of Life and Palliative Care, MS 260, 262 North Danny Thomas Place, Memphis, TN 38105. E-mail: Justin.Baker@stjude.org 
Levine et al

proving the quality of PC and end-of-life (EOL) care. The 1-year pilot program was successful, and in March 2008 the program was fully implemented, offering PC services throughout the institution. The program was designated as the Quality of Life Service (QoLS) to indicate that the team provided symptom management, care coordination, and advanced care planning for children with complex needs at any stage of illness.

The demographic characteristics of PPC programs $^{5}$ and the development of general PPC programs in inpatient and outpatient settings ${ }^{6-10}$ have been reported. However, the growth trajectory of a PC program in a comprehensive cancer center, particularly for pediatric patients, remains poorly described. The experience of establishing a PPC program and its successful integration over time in a comprehensive cancer center can serve as a model for other programs on a broad scale. In this study, we describe the 8-year experience of integrating PC with cancer care for pediatric patients at St. Jude, which exemplifies an evolving paradigm for comprehensive cancer care and can aid other health care institutions in establishing similar programs.

\section{Methods}

\section{Quality of Life Service}

The QoLS began as a pilot project on March 26, 2007, in the solid tumor and neuro-oncology services at St. Jude. St. Jude is a free-standing pediatric hospital, designated as a national comprehensive cancer center, dedicated to the care of children with cancer and select life-threatening illnesses. With 78 inpatient beds and numerous outpatient clinics, St. Jude serves children from a wide local catchment area, as well as patients referred from across the United States and around the world. The primary goal of the QoLS pilot was to demonstrate the feasibility of a service offering interventions designed to enhance advanced care planning, symptom control, care coordination, and EOL care for pediatric patients. The QoLS functioned on a consultative basis working together with the patients' primary teams in an effort to optimize collaborative care and maintain patient-provider relationships throughout the disease trajectory. The QoLS conducted comprehensive evaluations for individualized care, made observations and offered recommendations to the primary team for care planning and coordination, and activated measures to improve overall patient care. QoLS consults included advance care planning; symptom control; care coordination and continuity; emotional, social, and spiritual support; EOL care; and bereavement support, with care provided in inpatient, outpatient, and in home-based settings.

The consultative model used by the QoLS was the 3-step Individualized Care Planning and Coordination model comprising (1) establishment of a relationship, built on an understanding of the illness experience from the perspective of the patient and family, sharing relevant information, and assessing ongoing needs; (2) negotiation, including prognostication, establishing goals of care, and discussing treatment options; and (3) development of a mutually agreed-upon plan, including life and medical planning for the patient and family. ${ }^{14}$

The pilot demonstrated feasibility and highlevel family and staff satisfaction, and in 2008 the QoLS opened as a hospital-wide consult service available to all general clinical services at St. Jude. QoLS was staffed by 2 physicians, each at $50 \%$ clinical effort (1.0 clinical full time employee [FTE]) and 1 nurse practitioner. In addition to providing clinical care, the QoLS presented more than 200 educational offerings to hospital staff and trainees across the gamut in the institution and in the community, including nursing, child life, social work, chaplaincy, pharmacy, rehabiliation, medical students, residents, fellows, and hospice staff. The QoLS also partnered with medical and psychosocial providers in other divisions within the institution and with communitybased programs for expanded resources. With the tremendous growth of the service, in 2012 the team expanded to 2 physicians, 2 nurse practitioners, a full-time bereavement coordinator, an administrative assistant, and a clinical research associate (a total of 6 full FTEs). The service further expanded its reach by building a multi-institutional outpatient PC and hospice program, in which QoLS clinicians provide medical management and care coordination working in conjunction with community hospice personnel, to serve patients across care settings.

\section{Study Design and Participants}

A retrospective study was conducted of patients seen by the QoLS at St. Jude from the program's inception on March 26, 2007, through December 31, 2014. The study was approved by the St. Jude Institutional Review Board (IRB) as a quality im- 
provement project. Medical records of patients in the QoLS divisional database were cross-checked against the hospital-wide Health Information Management System's records to identify 615 patients seen at least once by the QoLS during this period. Data from electronic medical records were extracted, and a comprehensive password protected database was created to retrospectively evaluate and track the performance of the QoLS over time. The following information was obtained from the QoLS clinical notes: patient name, medical record number, date of birth, sex, race, referring service, diagnosis group, date of diagnosis, date first seen at St. Jude, QoLS encounter dates and types, goal of care at the time of initial consult (as documented in clinical notes in 1 of 4 categories based on presence and goal of diseasedirected therapy, including cure, prolong life with a focus on cure, prolong life with focus on comfort, and comfort only), code status, hospice enrollment, date of death, and location of death. There were no exclusion criteria, and the information from records of all 615 patients was used for the study.

\section{Statistical Analysis}

Simple frequencies, means, medians, standard deviations, percent comparisons, and trajectories were calculated for each variable. Descriptive statistics were generated from comparison of variables in the database and maintained in an excel spreadsheet, and calculations were made to determine trends. For time-dependent variables, only information from patients who had a minimum of 365 days of potential follow-up from date of initial QoLS consultation within the study period was included for statistical analysis. A maximum of 365 days was set to normalize data across years for meaningful comparison.

\section{Results}

Demographic information including age at initial QoLS consultation, race, sex, and diagnostic group is provided in Table 1. In the pilot year, QoLS consultations were exclusively offered to solid tumor and neuro-oncology patients, but with hospital-wide availability, the patients' diagnoses became a balanced representation over time.

The QoLS saw a dramatic increase in the number of new consults per year from 17 in its pilot year to 115 in 2014. The total number of patient encounters per year increased from 58 in its pilot year to 1,297 in
2014. The mean number of encounters per patient, by year of initial QoLS consultation, more than tripled from 5.1 in 2007 to 16.1 in 2014 (Figure 1).

In the early years of the hospital-wide QoLS service, the goal of care at initial consultation was more likely focused on "comfort only": 27\% (13 of 48) in 2008 and 37\% (23 of 62) in 2009. The goal of "comfort only" markedly decreased to $12 \%$ (12 of 100 ) and 13\% (15 of 115) in 2013 and 2014, respectively. Over time, the percentage of patients seen by the QoLS with a goal of cure at the initial QoLS consultation increased from 25\% (12 of 48) and 18\% (11 of 62 ) in 2008 and 2009, respectively, to 58\% (67 of 115 ) in 2014 (Figure 2).

Between 2008 and 2014, the percentage of patients not seen by the QoLS until they had been at St. Jude for more than 1 year was cut almost in half (from $58 \%$ to $31 \%$ ). Those whose initial QoLS consultation occurred within 1 month of their arrival increased approximately 6 -fold (from $4 \%$ to $23 \%$ ), those consulted within 3 months roughly tripled (from 13\% to 38\%), and those first seen within 6 months nearly doubled (from $27 \%$ to $52 \%$ ) (Figure 3 ). The median number of days between the date of initial consultation to the patient's date of death (or maximum 365 days) increased 4-fold from 52 days in 2008 (the first full QoLS calendar year) to 223 days in 2014 (Figure 4). Although a sustained trend over time for location of death was not observed, for deceased QoLS patients, an outpatient location of death became more prevalent after the pilot year, with $42 \%$ outpatient deaths in 2007 increasing to a majority in each subsequent year (range, 51\%-74\%; Figure 5). Data from deceased patients at St. Jude, available quarterly from April 2008, revealed a dramatic increase in the proportion of patients who received PC services before death, increasing from approximately $50 \%$ to nearly $100 \%$ for each quarter since 2012 (Figure 6).

\section{Discussion}

Since the inception of the QoLS, the benefits of integrating PC services into the ongoing cancer treatment of patients were evident. Over the years, an increase was seen in the use of PC services, reflecting a growing culture of acceptance and incorporation of PC services and resulting in the proportionate use of the QoLS by all clinical services. The culture shift and growing acceptance of PC at St. Jude was 
Levine et al

\begin{tabular}{|c|c|c|c|c|c|c|c|c|}
\hline & $\begin{array}{l}2007 \\
\left(n^{a}=17\right)\end{array}$ & $\begin{array}{l}2008 \\
(n=48)\end{array}$ & $\begin{array}{l}2009 \\
(n=62)\end{array}$ & $\begin{array}{l}2010 \\
(n=76)\end{array}$ & $\begin{array}{l}2011 \\
(n=71)\end{array}$ & $\begin{array}{l}2012 \\
(n=126)\end{array}$ & $\begin{array}{l}2013 \\
(n=100)\end{array}$ & $\begin{array}{l}2014 \\
(n=115)\end{array}$ \\
\hline \multicolumn{9}{|l|}{ Age at consult, y } \\
\hline Mean & 10.0 & 11.8 & 9.6 & 10.4 & 10.9 & 10.6 & 8.8 & 9.7 \\
\hline Median & 9.6 & 11.6 & 8.9 & 9.0 & 10.3 & 10.3 & 7.6 & 8.7 \\
\hline Range & $0.9-25.4$ & $0.1-24.4$ & $0.3-22.6$ & $0-28.4$ & $0.1-23.6$ & $0-23.8$ & $0.7-22.6$ & $0.5-23.9$ \\
\hline \multicolumn{9}{|l|}{ Race, n (\%) } \\
\hline White & $9(52.94 \%)$ & $29(60.42 \%)$ & $37(59.68 \%)$ & $54(71.05 \%)$ & $48(67.61 \%)$ & $83(65.87 \%)$ & $61(61 \%)$ & $77(66.96 \%)$ \\
\hline Black & $6(35.29 \%)$ & $16(33.33 \%)$ & $18(29.03 \%)$ & $18(23.68 \%)$ & $15(21.13 \%)$ & $28(22.22 \%)$ & $25(25 \%)$ & $24(20.87 \%)$ \\
\hline Other & $2(11.76 \%)$ & $3(6.3 \%)$ & $7(11.3 \%)$ & $4(5.3 \%)$ & $8(11.3 \%)$ & $15(11.9 \%)$ & $14(14 \%)$ & $14(12.2 \%)$ \\
\hline \multicolumn{9}{|l|}{ Sex, $\mathrm{n}(\%)$} \\
\hline Male & $7(41.18 \%)$ & $31(64.58 \%)$ & $32(51.61 \%)$ & $40(52.63 \%)$ & $48(67.61 \%)$ & $77(61.11 \%)$ & $51(51 \%)$ & $66(57.39 \%)$ \\
\hline Female & $10(58.82 \%)$ & $17(35.42 \%)$ & $30(48.39 \%)$ & $36(47.37 \%)$ & $23(32.39 \%)$ & 49 (38.89\%) & $49(49 \%)$ & $49(42.61 \%)$ \\
\hline \multicolumn{9}{|c|}{ Diagnostic group, $\mathrm{n}(\%)^{\mathrm{b}}$} \\
\hline Solid tumor & $5(29 \%)$ & $10(21 \%)$ & $23(37 \%)$ & $22(29 \%)$ & $21(30 \%)$ & $40(32 \%)$ & $30(30 \%)$ & $46(40 \%)$ \\
\hline Hematologic & $0(0 \%)$ & $18(38 \%)$ & $17(27 \%)$ & $15(20 \%)$ & $22(31 \%)$ & $44(35 \%)$ & $31(31 \%)$ & $33(29 \%)$ \\
\hline $\begin{array}{l}\text { Neuro- } \\
\text { Oncologic }\end{array}$ & $12(71 \%)$ & $20(42 \%)$ & $22(35 \%)$ & $37(49 \%)$ & $27(38 \%)$ & $42(33 \%)$ & $38(38 \%)$ & $36(31 \%)$ \\
\hline BMT & $0(0 \%)$ & $9(19 \%)$ & $10(16 \%)$ & $9(14 \%)$ & $12(17 \%)$ & $28(22 \%)$ & $20(20 \%)$ & $25(22 \%)$ \\
\hline
\end{tabular}

an represents the number of initial Quality of Life Services consultations in each calendar year.

bDiagnostic groups were determined by primary diagnosis and referring service. Percentages may exceed $100 \%$ as bone marrow transplant (BMT) recipients

are also categorized by their underlying diagnoses.

a deliberate process over years of fostering positive relationships and collaborations, as well as actively avoiding conflict, to demonstrate the collegial and complementary nature of the QoLS. The increase in the number of patients from varying diagnosis groups seen by the QoLS over the 8-year period led to a more balanced representation of the St. Jude patients, which supports the success of early interdisciplinary team-building efforts.

Along with this hospital-wide culture shift came an increasing demand to integrate $\mathrm{PC}$ into the treatment plan of patients at St. Jude, which led to a dramatic increase in the number of new QoLS consults each year. Similar findings have been reported in adult patients with cancer, with PC referrals steadily increasing over time and reflecting a general adoption of PC as a beneficial service for patients and physicians. ${ }^{16,17}$ However, in adult patients with cancer, there were significant differences in the use of PC among various clinical services, which suggested that symptom burden was underestimated in certain diagnostic groups. ${ }^{17}$ Although the underestimation of symptom burden and underutilization of PC ser- vices might also occur in pediatric patients with cancer, and the effect of PC services on symptom burden is outside the scope of this study, the proportionate use of the QoLS by all clinical services at our institution is a reassuring finding.

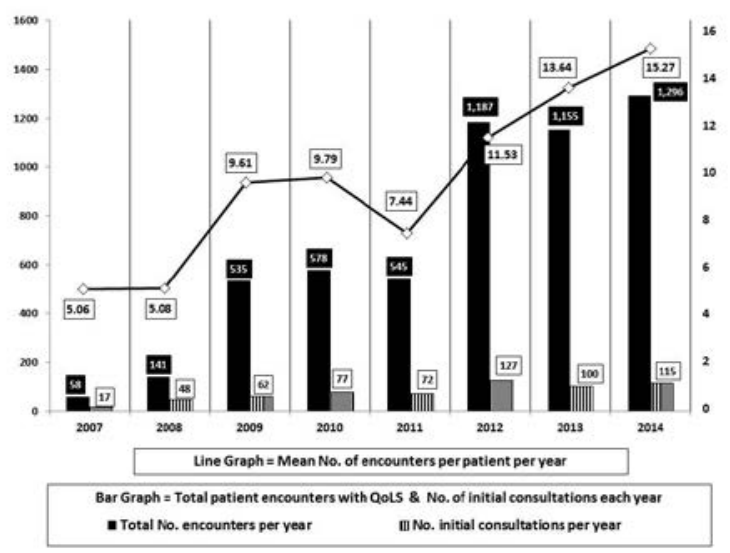

Figure 1. Number of initial Quality of Life Service (QoLS) consultations per year, total QoLS patient encounters per year, and mean number of QoLS encounters per patient by year of the patient's initial QoLS consultation (March 2007-December 2014).

Note: the mean number of encounters per patient for 2014 includes only those patients whose initial QoLS consultation occurred between January 1, 2014, and September 30, 2014, to allow for 365 days of potential follow-up before death. Data for all the other years include all patients seen that year. 


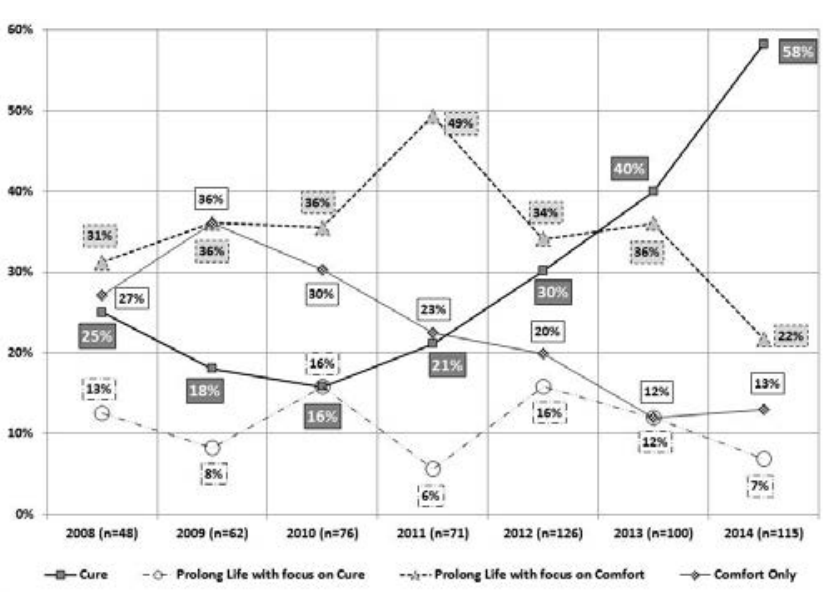

Figure 2. Goals of care for patients at initial Quality of Life Service (QoLS) consultation (January 2008-December 2014).

Cure: receiving disease directed therapy with realistic chance of cure; Prolong life with focus on cure: receiving disease directed therapy with goal disease stabilization and less likely chance of cure; Prolong life with focus on comfort: receiving disease-directed palliative therapy for life prolongation without curative intent; Comfort only: receiving palliative therapy for symptom management only.

Note: $\mathrm{n}$ represents the number of initial QoLS consultations in each calendar year.

There was also a striking increase in the total number of patient encounters per year by the QoLS. This finding indicates that the QoLS not only saw more patients but also followed them more regularly and for longer periods. The increase each year in the median number of encounters per patient further substantiates that patients were seen by QoLS with increasing regularity and sustained continuity from the time of consultation.

A trend toward earlier consultation was supported by the shift in the goal of care at initial QoLS consultation, the longer duration of survival from the time of initial QoLS consultation, and the QoLS consultation occurring closer to the time of arrival at St. Jude over the years. The culture shift at St. Jude since 2008 likely contributed to the earlier referral of patients to the QoLS by the primary services. In the earlier years of the service, more patients were seen with the goal of comfort if further disease-directed therapy was no longer an option, yet in later service years, more patients were referred while pursuing the goal of cure or the prolongation of life for concurrent PC and disease-directed therapy. In contrast to observations on PC consultations for adult patients with cancer which showed a decrease over time in the average number of days from the first consultation to death, ${ }^{17}$ our observed trend toward increased duration of survival from initial consultation further

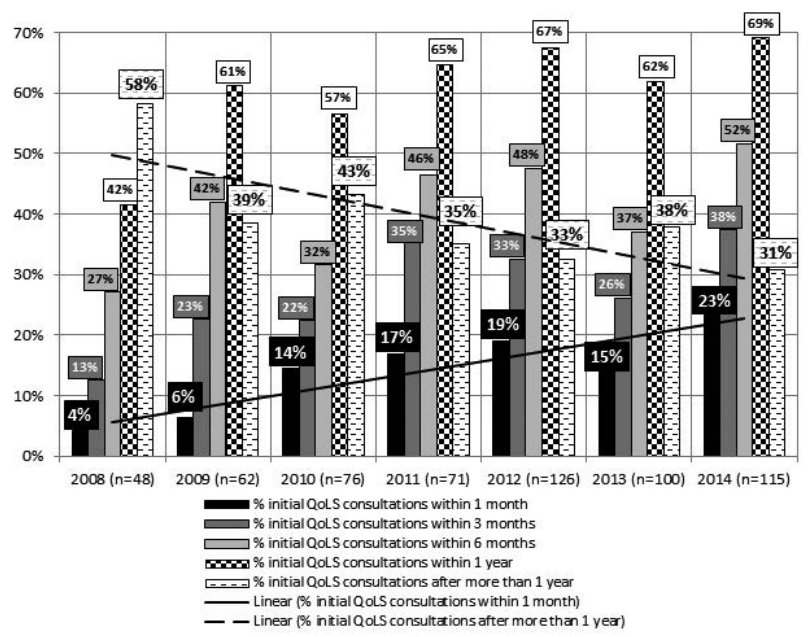

Figure 3. Demonstration of earlier palliative care involvement over time by way of length of time between the date first seen at St. Jude and date of initial Quality of Life Service (QoLS) consultation (January 2008-December 2014).

Note: $\mathrm{n}$ represents the number of initial QoLS consultations in each calendar year.

highlights the earlier involvement of the QoLS in the patients' disease trajectory over time.

Early integration of PC into cancer care has been touted as the optimal model and can improve patient quality of life and caregiver satisfaction, and, as observed in patients with metastatic non-smallcell lung cancer, may increase the duration of survival. ${ }^{18}$ The demonstrated benefits of early PC integration into cancer care led ASCO to recommend PC services for all high-risk oncology patients and for increased research in this field. ${ }^{19}$ Despite these evidence-based recommendations for early integration of PC in cancer care, ${ }^{20}$ adult and pediatric oncologists refer only a small proportion of patients to PC services, typically late in the disease course when the goal of cure is no longer an option and patients are often at the EOL..$^{21,22}$ In contrast, our data from the QoLS at St. Jude indicate a trend toward earlier consultation in a growing number of patients, allowing for potentially improved quality of care through PC integration.

After the pilot year, there was also a shift in QoLS patients' location of death from primarily the inpatient setting to an increased prevalence in an outpatient setting. Patient characteristics such as goal of care, complexity of care, and patient acuity are key factors that can influence location of death, such as the observed number per year of intensive care unit deaths, but these factors were unable to be determined in our study, therefore limiting the 


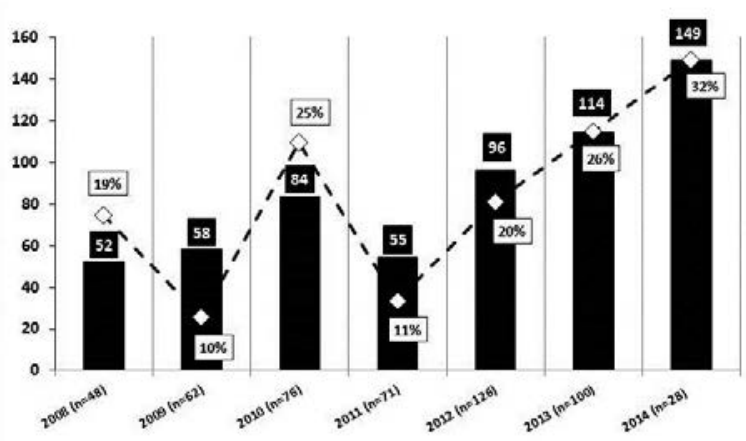

Line Graph $=\%$ of patients who survived 365 days after date of inital QoLs consultation Bar Graph=Median Number of days to date of death from date of initial QoLs consultation or 365 days

Figure 4. Patients' survival duration from initial Quality of Life Service (QoLS) consultation (January 2008-December 2014).

Note: $\mathrm{n}$ represents the number of initial QoLS consultations in each calendar year. The 2014 analysis includes only those patients whose initial QoLS consultation occurred between January 1, 2014, and September 30, 2014, to allow for 365 days of potential follow-up before death. Data from all the other years include all patients seen that year. The maximum follow-up for all patients each year was set to 365 days in order to normalize data for comparison.

conclusions with regard to this data. Further, the preferred location of death, and the ability to accommodate this preference, was not known because this information was often not available from the medical records. Delineating the preferred location of death and the ability to meet these preferences is an area for future investigation. Studies show that many patients and families prefer an outpatient location of death when feasible. ${ }^{23}$ Although we could not determine the preferred location of death, increased outpatient location of death in our study after the pilot year suggests that increased availability of PC services enabled outpatient EOL care and a greater ability to meet the preference of many patients and families.

A key quality indicator, as outlined by the $\mathrm{Na}$ tional Quality Forum and the Center to Advance Palliative Care, is the hospital-wide access to PC services for patients. A remarkable observation in our study was the hospital-wide shift in the receipt of PC services before death for patients. Although not all deceased patients had been actively followed by the QoLS, hospital-wide almost all patients had received PC services before death since 2012. This increase coincided with expansion of the QoLS staff in 2012, the inception of a collaborative home-based $\mathrm{PC}$ and hospice program, increased interdisciplinary collaboration, a large number of wide-reaching educational efforts by the QoLS, and implementation by the QoLS of institutional standard operating proce-

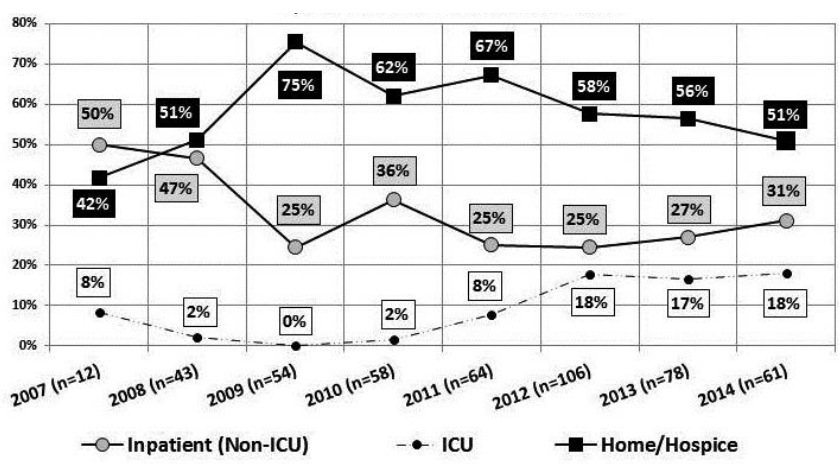

Figure 5. Location of death of patients for whom the Quality of Life Service (QoLS) provided consultation (March 2007-December 2014). Abbreviation: ICU, intensive care unit.

Note: $n$ represents the number of initial QoLS consultations in each calendar year.

dures for patients nearing EOL, which all aided in changing the attitudes toward PC and improving the provision of $\mathrm{PC}$ services at the institution and beyond. The standard operating procedures for patients at EOL activates an interdisciplinary team meeting led by the QoLS aimed at enhancing comprehensive communication and collaboration among all medical providers involved in the care of the patient and ensuring that standards of preferred practices for palliative and EOL care according to the National Quality Forum are met. This facilitates proactive and comprehensive communication among PPC providers, the primary medical team, subspecialists, and home-based medical providers, which is essential to meeting the needs of patients nearing EOL.

One barrier to integrating PC services early into cancer care is the relative paucity of $\mathrm{PC}$ services across the United States, especially in pediatric facilities. ${ }^{24}$ The increasing demand for PC services led to the expansion of the QoLS resources and reach to increase PPC expertise and availability, including expanded QoLS clinical and research staff, a hospice and palliative medicine fellowship training program, and the creation of a home-based PC and hospice program in conjunction with the local children's hospital and community hospice. Weekly multidisciplinary team meetings were initiated with psychosocial and medical service providers in other divisions of the hospital to coordinate the care for all patients seen by the QoLS, improve communication among services, and focus on providing collaborative care encompassing the domains of physical, emotional, social, and spiritual care. For example, the QoLS staff did not include a dedi- 


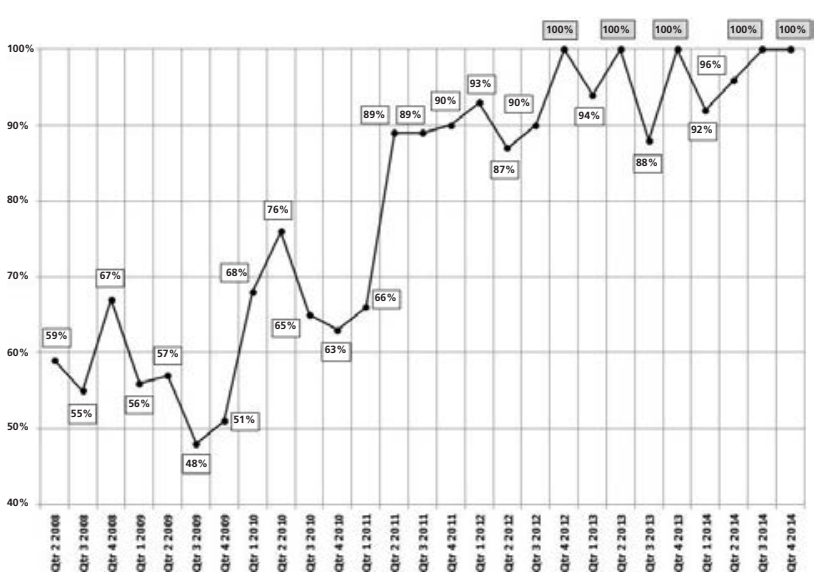

Figure 6. Percentage per quarter of deceased St. Jude patients who received palliative care services before death (Quarter 2, 2008-Quarter 4, 2014).

cated psychosocial provider but rather worked in conjunction with existing social workers, psychologists, child life specialists, and chaplains in St. Jude and in the community to provide comprehensive care. Expanding the resources, partnerships with other divisions, and educational efforts institutionally, as well as fostering partnerships with other local institutions for community resources, helped provide enhanced care for St. Jude patients across care settings and is essential for the success of any PPC program. Similarly, in many settings where PPC-trained providers and/ or resources are not abundant, institutions can use existing resources to meet the needs of their patients, with an expert PPC provider guiding coordination of care rather than personally delivering services.

Our study was limited by its retrospective nature of data over the course of 8 years. Thus, there might be incomplete data because the medical record format and templates, as well as documenting providers, changed over time. Further, documentation from patient encounters could have been omitted by clinicians at various time points, leading to incorrect data calculations and conclusions. Year 1 of the study (2007) was an incomplete calendar year and only included the pilot population of solid tumor and neuro-oncology. Data from year 8 (2014) were incomplete for time-dependent variables, because the passage of time for patients in the later months of 2014 was not sufficient to make comparisons with data from previous years.

Despite these limitations, the findings presented herein are novel and compelling and can be used to draw useful conclusions. The generalizability of our results might be limited because this is a singleinstitution study and St. Jude is a unique institutional model, but the PC model described herein and our findings on the QoLS over an 8-year period can guide other institutions in creating and integrating a PC service with wide applicability.

\section{Conclusions}

Since its inception in 2007, the QoLS at St. Jude has experienced a dramatic increase in the number of referrals and the total number of patient encounters, proportionate referrals by all clinical services, a trend toward earlier consultation and regular longer-term follow-up, a trend toward an increased outpatient location of death, and the near-universal involvement of PPC at the EOL. What started as a pilot of a fledgling PPC consult service has blossomed into a fully resourced consultative service that extends care across multiple settings to patients at various stages of illness, and an educational program that expands the base of knowledge and expertise locally and nationally to promote quality care at the institution and beyond. The St. Jude QoLS has emerged as a paradigm of successful PC implementation and integration in a comprehensive cancer center and can serve as a model for other health care institutions.

\section{References}

1. Kane JR, Himelstein BP. Palliative care for children. In: Berger AM, Shuster JL, Von Roenn JH, eds. Principles and Practice of Palliative Medicine and Supportive Oncology, 3rd ed. Philadelphia, PA: Lippincott Williams \& Wilkins; 2007:1044-1061.

2. Vickers JL, Carlisle C. Choices and control: parental experiences in pediatric terminal home care. J Pediatr Oncol Nurs 2000;17:12-21.

3. Davies B, Deveau E, deVeber B, et al. Experiences of mothers in five countries whose child died of cancer. Cancer Nurs 1998;21:301-311.

4. Field MJ, Behrman RE, eds. When children die: improving palliative and end-of-life care for children and their families. Institute of Medicine (U.S. Committee on Palliative and End-of-Life Care for Children). Washington, DC: National Academies Press; 2003.

5. Kang TI, Feudtner C. Advances in pediatric palliative medicine in the United States. Prog Palliat Care 2012;20:331-336.

6. Ward-Smith P, Linn JB, Korphage RM, et al. Development of a pediatric palliative care team. J Pediatr Health Care 2007;21:245-249.

7. Schmidt K. Pediatric palliative care: starting a hospital-based program. Pediatr Nurs 2011;37:268-274.

8. Pelant D, McCaffrey T, Beckel J. Development and implementation of a pediatric palliative care program. J Pediatr Nurs 2012;27:394-340.

9. Kost-Byerly $S$, Chalkiadis G. Developing a pediatric pain service. Paediatr Anaesth 2012;22:1016-1024

10. Hubble RA, Ward-Smith $P$, Christenson $K$, et al. Implementation of a palliative care team in a pediatric hospital. J Pediatr Health Care 2009;23:126-131.

11. American Academy of Pediatrics. Committee on Bioethics and Committee on Hospital Care. Palliative care for children. Pediatrics 2000;106(2 Pt 1):351-357. 


\section{Levine et al}

12. Field M, Cassel CK, eds. Approaching Death: Improving Care at the End of Life. Washington, DC: National Academy Press; 1997.

13. Johnson LM, Snaman JM, Cupit MC, Baker JN. End-of-life care for hospitalized children. Pediatr Clin North Am 2014;61:835-854.

14. Baker JN, Barfield R, Hinds PS, Kane JR. A process to facilitate decision making in pediatric stem cell transplantation: the individualized care planning and coordination model. Biol Blood Marrow Transplant 2007;13:245-254.

15. Harper J, Hinds PS, Baker JN, et al. Creating a palliative and end-of-life program in a cure-oriented pediatric setting: the zig-zag method. J Pediatr Oncol Nurs 2007;24:246-254.

16. Homsi J, Walsh D, Nelson KA, et al. The impact of a palliative medicine consultation service medical oncology. Support Care Cancer 2002;10:337342.

17. Dhillon N, Kopetz S, Pei BL, et al. Clinical findings of a palliative care consultation team at a comprehensive cancer center. J Palliat Med 2008;11:191-197.

18. Temel J, Greer J, Muzikansky A, et al. Early palliative care for patients with metastatic non-small-cell lung cancer. N Engl J Med 2010;363:733-742.
19. Smith TJ, Temin S, Alesi ER, et al. American Society of Clinical Oncology provisional clinical opinion: the integration of palliative care into standard oncology care. J Clin Oncol 2012;30:880-887.

20. Bauman J, Temel J. The integration of early palliative care with oncology care: the time has come for a new tradition. J Natl Compr Canc Netw 2014;12:1763-1771.

21. Wentlandt K, Krzyzanowska MK, Swami N, et al. Referral practices of pediatric oncologists to specialized palliative care. Support Care Cancer 2014;22:15-22.

22. Johnston D, Vadeboncoeur C. Palliative care consultation in pediatric oncology. Support Care Cancer 2012;20:799-803.

23. Bluebond-Langner M, Beecham E, Candy B, et al. Preferred place of death for children and young people with life-limiting and life-threatening conditions: a systematic review of the literature and recommendations for future inquiry and policy. Palliat Med 2013;27:705-713.

24. Johnston DL, Nagel K, Friedman DL, et al. Availability and use of palliative care and end-of-life services for pediatric oncology patients. J Clin Oncol 2008;26:4646-4650. 